\title{
Optimal Index Assignment for Multiple Description Lattice Vector Quantization (Extended Abstract)
}

\author{
Xiang Huang and Xiaolin $\mathrm{Wu}$ \\ Department of Electrical \& Computer Engineering \\ McMaster University, Canada, L8S 4L8 \\ huangx4@mcmaster.ca; xwu@ece.mcmaster.ca
}

\begin{abstract}
Optimal index assignment of multiple description lattice vector quantizer (MDLVQ) can be posed as a large-scale linear assignment problem. But is this expensive algorithmic approach necessary? This paper presents a surprisingly simple index assignment algorithm for high-resolution MDLVQ of $K \geq 2$ balanced descriptions in any dimensions. Despite its simplicity, the new algorithm is optimal for a large family of lattices encountered in theory and practice, in terms of minimizing the expected distortion for any side description loss rate and any side entropy rate. This work offers exact combinatoric constructions of optimal index assignments, rather than arguing for the optimality asymptotically. Consequently, the optimality holds for all values of sublattice index $N$ (i.e., over all trade-offs between the central and side distortions), rather than for very large $N$ only. Furthermore, the time complexity of the new algorithm is $O(N)$ as opposed to $O\left(N^{6}\right)$ for a current linear assignment-based method.

New and improved closed form expressions of the expected distortion as the function of $N$ and $K$ are also presented. Thus the optimal values of $N$ and $K$ can be computed.
\end{abstract}

\section{Introduction}

An effective MDC technique is multiple description vector quantization (MDVQ). Unfortunately, optimal MDVQ design is computationally intractable (optimal singledescription VQ design is already NP-hard) unless NP equals P. To overcome this operational difficulty, one typically imposes some structure on codebooks. A popular structured MDVQ approach is multiple description lattice vector quantization (MDLVQ). Given a lattice $\Lambda$ for central description, the problem of optimal code design reduces to one of associating $\Lambda$ to a so-called sublattice $\Lambda_{s}$ (typically $\Lambda_{s} \subset \Lambda$ ), and establishing a one-to-one mapping, called index assignment $\alpha$, between a point $\lambda \in \Lambda$ and an ordered $K$-tuple $\left(\lambda_{1}, \ldots, \lambda_{K}\right) \in \Lambda_{s}^{K}$, where $K$ is the number of side descriptions [2]. The goal of the MDLVQ index assignment is to find the bijection function $\alpha: \Lambda \leftrightarrow \alpha(\Lambda) \subset \Lambda_{s}^{K}$ that minimizes an expected distortion measure over all subsets of $K$ descriptions. 
Vaishampayan et al. discussed a "guiding principle" for constructing an optimal MDLVQ index assignment [3]. Some example index assignments were presented without the proof of their optimality. More recently, Ostergaard et al. studied the problem of optimal MDLVQ index assignment, and presented some asymptotical results [4]. But to our best knowledge, the only known exact solution of optimal MDLVQ index assignment is a linear assignment algorithm of extremely high complexity [4] $\left(O\left(N^{6}\right)\right.$ as shown in Section 3.4). In this paper we propose a very simple linear-time algorithm for MDLVQ index assignment for balanced descriptions, and then prove, under the assumption of high resolution, that the algorithm is optimal for many commonly used good lattices, over the entire range of achievable central distortions given the side entropy rate. The optimality is in terms of minimizing the expected distortion given the side description loss rate and given the side entropy rate.

This extended abstract is organized as follows. The next section formulates the problem and introduces necessary notations. Section 3 presents and analyzes the new index assignment algorithm, together with some insights that lead us to the algorithm. Section 4 proves the optimality of the new index assignment under a condition of the so-called S-similarity of sublattices. Section 5 shows that the S-similarity can be satisfied by lattices commonly used in signal quantization. Sections 6 and 7 present some new and improved closed form expressions of the expected distortion for optimal MDLVQ, which allow the optimal parameters $N$ and $K$ to be computed.

\section{Problem Formulation and Notations}

In an MDLVQ system of $K$ descriptions, an input vector $x \in R^{L}$ is first quantized to the nearest lattice point $\lambda$ in a lattice $\Lambda \subset R^{L}$, called central (fine) lattice. Then the point $\lambda$ is mapped by a bijective labeling function $\alpha: \Lambda \leftrightarrow \Lambda_{s}^{K}$ to an ordered $K$-tuple $\left(\lambda_{1}, \lambda_{2}, \cdots, \lambda_{K}\right)$, where $\Lambda_{s}$ is a coarse lattice. Typically, $\Lambda_{s} \subset \Lambda$, hence $\Lambda_{s}$ is called a sublattice. Let the components of $\alpha$ be $\left(\alpha_{1}, \alpha_{2}, \cdots, \alpha_{K}\right)$, i.e., $\alpha_{k}(\lambda)=\lambda_{k}, 1 \leq k \leq K$. With the function $\alpha$ the encoder generates $K$ descriptions of $x: \lambda_{k}, 1 \leq k \leq K$, and transmits each description via an independent channel to a receiver.

If the decoder receives all $K$ descriptions, it can reconstruct $x$ to $\lambda$ with the inverse labeling function $\alpha^{-1}$. In this best case the average distortion per dimension is given by

$$
d_{c}=\sum_{\lambda \in \Lambda} \int_{V(\lambda)}\|x-\lambda\|^{2} p_{X}(x) d x .
$$

where $V(\lambda)$ is the Voronoi cell of the lattice point $\lambda \in \Lambda$.

In general, due to channel losses, the decoder receives only a subset $\chi$ of the $K$ descriptions, and it can reconstruct $x$ to the average of the received descriptions:

$$
\hat{x}=\frac{1}{|\chi|} \sum_{\lambda_{i} \in \chi} \lambda_{i} .
$$

Assuming that the $K$ channels are independent, and each has a probability $p_{l}$ to fail, we can write the expected distortion as

$$
D=\sum_{k=0}^{K}\left(\begin{array}{c}
K \\
k
\end{array}\right)\left(1-p_{l}\right)^{k} p_{l}^{K-k} D_{k}
$$


where $D_{k}$ is the average distortion when receiving $k$ out of $K$ descriptions. For lattices of high resolution or uniform source distribution $p_{X}$ such that $\lambda$ is the centroid of its Voronoi region, one can show [4]

$$
D=\left(1-p_{l}^{K}\right) d_{c}+\sum_{\lambda \in \Lambda}\left(\zeta_{1}\left\|\lambda-m_{K}\right\|^{2}+\zeta_{2} \frac{1}{K} \sum_{k=1}^{K}\left\|\lambda_{k}-m_{K}\right\|^{2}\right) P(\lambda)+p_{l}^{K} E\left[\|X\|^{2}\right],
$$

where

$$
\zeta_{1}=\sum_{k=1}^{K-1}\left(\begin{array}{c}
K \\
\kappa
\end{array}\right)\left(1-p_{l}\right)^{k} p_{l}^{K-k}, \quad \zeta_{2}=\sum_{k=1}^{K-1}\left(\begin{array}{c}
K \\
k
\end{array}\right)\left(1-p_{l}\right)^{k} p_{l}^{K-k} \frac{K-k}{(K-1) k},
$$

$P(\lambda)=\operatorname{Pr}(Q(X)=\lambda)$, and $m_{K}$ is the centroid of all $K$ descriptions $m_{K} \equiv \frac{1}{K} \sum_{k=1}^{K} \lambda_{k}$. Now note that $d_{c}, p_{l}, \zeta_{1}$ and $\zeta_{2}$ are constant given source and channel statistics and given $\Lambda, \Lambda_{s}$. We reduce the problem of optimal MDLVQ index assignment to that of minimizing

$$
d_{s} \equiv \sum_{\lambda \in \Lambda}\left(\frac{1}{K} \sum_{i=1}^{K}\left\|\lambda_{i}-m_{K}\right\|^{2}+\frac{\zeta_{1}}{\zeta_{2}}\left\|\lambda-m_{K}\right\|^{2}\right) P(\lambda)
$$

\section{Index Assignment Algorithm}

This section presents a very simple index assignment algorithm for MDLVQ of $K$ balanced descriptions. For clarify of presentation and avoiding immaterial details in describing our main ideas, we consider the sublattices $\Lambda_{s}$ that are geometrically similar to $\Lambda$ and clean (no central lattice point lies on the boundary of a sublattice Voronoi cell). Let us first reveal some useful lattice structures to be exploited by the index assignment algorithm.

\subsection{Useful lattice properties}

In the following study of optimal index assignment for $K$ balanced descriptions, the lattice $\Lambda_{s / K} \equiv \frac{1}{K} \Lambda_{s}$ plays an important role, and it has the following interesting relations to $\Lambda$ and $\Lambda_{s}$.

Property $1 m\left(\lambda_{1}, \lambda_{2}, \cdots, \lambda_{K}\right)=\frac{1}{K} \sum_{k=1}^{K} \lambda_{k}$ is an onto (but not one-to-one) map: $\Lambda_{s}^{K} \rightarrow \Lambda_{s / K}$.

Proof: 1) $\left.\lambda_{1}, \lambda_{2}, \cdots, \lambda_{K} \in \Lambda_{s} \Rightarrow \sum_{k=1}^{K} \lambda_{k} \in \Lambda_{s} \Rightarrow \frac{1}{K} \sum_{k=1}^{K} \lambda_{k} \in \Lambda_{s / K} ; 2\right)$ $\forall \tau \in \Lambda_{s / K}$, let $\lambda_{1}=K \tau, \lambda_{2}=\cdots=\lambda_{K}=0$, then $\lambda_{1}, \lambda_{2}, \cdots, \lambda_{K} \in \Lambda_{s}$ and $m\left(\lambda_{1}, \lambda_{2}, \cdots, \lambda_{K}\right)=\tau$.

This means that the centroid of any $K$-tuples in $\Lambda_{s}^{K}$ must be in $\Lambda_{s / K}$, and further $\Lambda_{s / K}$ consists only of these centroids.

The sublattice $\Lambda_{s / K}$ partitions the space into Voronoi cells. Denote the Voronoi cell of a point $\tau \in \Lambda_{s / K}$ by

$$
V_{s / K}(\tau)=\left\{x:\|x-\tau\| \leq\|x-\tilde{\tau}\|, \forall \tilde{\tau} \in \Lambda_{s / K}\right\} .
$$


We note that no central lattice point of $\Lambda$ lies on the boundary of any Voronoi cell of $\Lambda_{s / K}$.

Property $2 \Lambda_{s / K}$ is clean, if $\Lambda_{s}$ is clean.

Proof: Assume for a contradiction that there was a point $\lambda \in \Lambda$ on the boundary of $V_{s / K}(\tau)$ for a $\tau \in \Lambda_{s / K}$. Scaling both $\lambda$ and $V_{s / K}(\tau)$ by $K$ places $K \lambda$ on the boundary of $K V_{s / K}(\tau)=\left\{K x:\|K x-K \tau\| \leq\|K x-K \tilde{\tau}\|, \forall \tilde{\tau} \in \Lambda_{s / K}\right\}$. But $K \lambda$ is a point of $\Lambda$, and $K V_{s / K}(\tau)$ is nothing but the Voronoi cell $V_{s}$ of the sublattice point $K \tau \in \Lambda_{s}$, or the point $K \lambda \in \Lambda$ lies on the boundary of $V_{s}(K \tau)$, contradicting that $\Lambda_{s}$ is clean.

Property 3 Both lattices $\Lambda_{s}$ and $\Lambda$ are symmetric about any point $\tau \in \Lambda_{s / 2}$.

Proof: $\forall \tau \in \Lambda_{s / 2}$, we have $2 \tau \in \Lambda_{s}$, so $2 \tau-\lambda_{s} \in \Lambda_{s}$ holds for $\forall \lambda_{s} \in \Lambda_{s}$; similarly, $\forall \tau \in \Lambda_{s / 2}$, we have $2 \tau \in \Lambda$, so $2 \tau-\lambda \in \Lambda$ holds for $\forall \lambda \in \Lambda$.

\subsection{Greedy labeling}

We are now ready to state a new index assignment algorithm. We partition the space by Voronoi cells at the sites of all points in $\Lambda_{s / K}$. According to Property 2, no point $\lambda \in \Lambda$ is on the boundary of any Voronoi region of $\Lambda_{s / K}$. To label the central lattice points inside $V_{s / K}(\tau)$, we choose the $\left|\Lambda \cap V_{s / K}(\tau)\right|$ nearest ordered $K$-tuples of sublattice points that have the same centroid $\tau$.

Interestingly, it follows from (3) that any bijective mapping between the $\mid \Lambda \cap$ $V_{s / K}(\tau) \mid$ center lattice points and the $\left|\Lambda \cap V_{s / K}(\tau)\right| K$-tuples yields the same value of $d_{s}$. One can choose an arbitrary assignment between the two sets and still minimize $d_{s}$.

Since there are $K^{L}$ classes of $\Lambda_{s / K} / \Lambda_{s}$, there are $K^{L}$ classes of $V_{s / K}(\tau)$. We only need to label one representative out of each class, and cover the whole space by shifting. Thus it suffices to label a total of $N$ central lattice points.

For the two description case, these $\left|\Lambda \cap V_{s / 2}(\tau)\right|$ ordered pairs are formed by the $\left|\Lambda \cap V_{s / 2}(\tau)\right|$ nearest sublattice points to $\tau$ in $\Lambda_{s}$ by Property 3. Note when $\tau \in \Lambda_{s}$, the ordered pair $(\tau, \tau)$ should be used to label $\tau$ itself.

\subsection{Two Examples}

Let us see how the proposed index assignment algorithm works via examples on an $A_{2}$ lattice, which are presented graphically. The $A_{2}$ lattice $\Lambda$ is generated by basis vectors represented by complex numbers: 1 and $\omega=1 / 2+i \sqrt{3} / 2$. The first example is a twodescription case, with the sublattice $\Lambda_{s}$ given by basis vectors $5-\omega, \omega(5-\omega)$, which is geometrically similar to $\Lambda$, has index $N=31$ and is clean (refer to Fig. 1). There are two kinds of Voronoi cells of $\Lambda_{s / 2}$, as shown by the solid and dashed boundaries in Fig. 1. The solid cell is centered at a central lattice point and contains 7 central lattice points. The dashed cell is centered at the midpoint of the line segment $O A$, and contains 8 central lattice points. To label the 7 central lattice points in $V_{s / 2}(O)$, we use the 7 nearest sublattice points to $O:(O, A, B, C, D, E, F)$. They form 6 ordered pairs with 


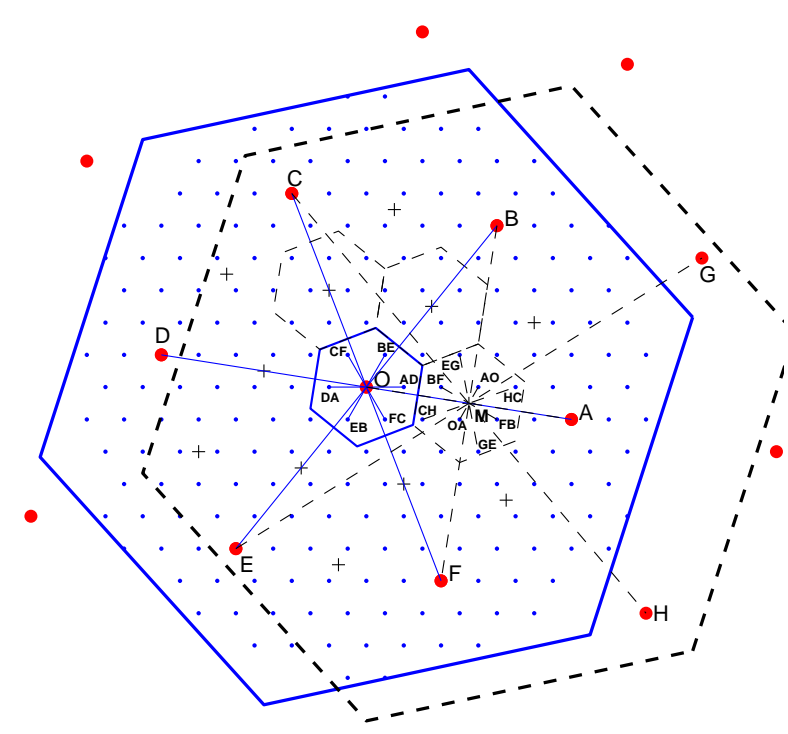

Figure 1: Two description index assignment for the $A_{2}$ lattice with index $N=$ 31. Points of $\Lambda, \Lambda_{s}$ and $\Lambda_{s / 2}$ are marked by $\cdot, \bullet$ and + , respectively.

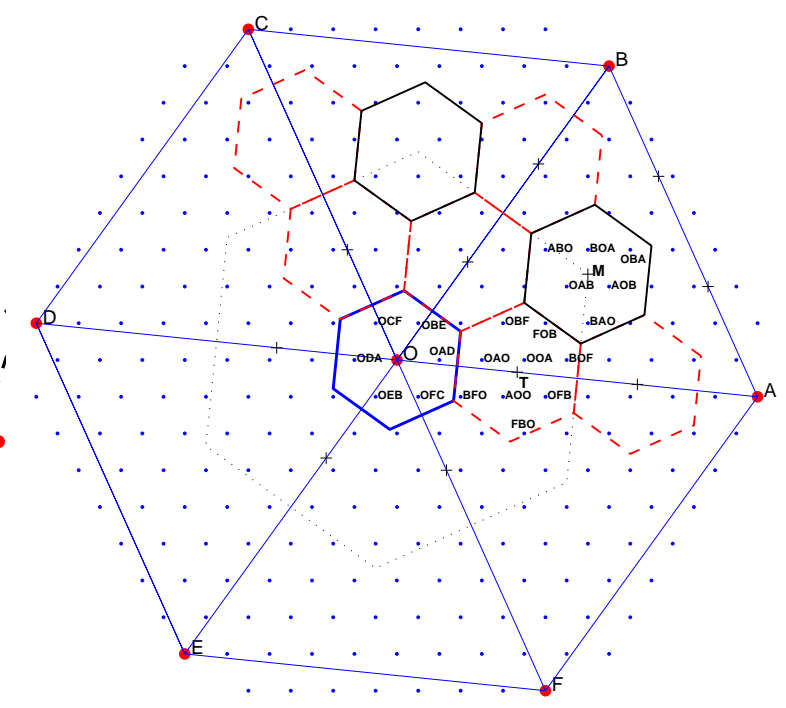

Figure 2: Three description index assignment for the lattice $A_{2}$ with index $N=73$. Points of $\Lambda, \Lambda_{s}$ and $\Lambda_{s / 3}$ are marked by $\cdot, \bullet$ and + , respectively.

the midpoint $O:((A, D),(D, A),(B, E),(E, B),(C, F),(F, C)$, and an unordered pair $(O, O)$ since $O$ is itself a sublattice point. To label the 8 central lattice points in $V_{s / 2}(M)$, we use the 8 nearest sublattice points to $M$ : $(O, A, B, F, C, H, E, G)$. They form 8 ordered pairs with midpoint $M:(O, A),(A, O),(B, F),(F, B),(C, H),(H, C)$, $(E, G),(G, E)$. The labels of those 7 and 8 central lattice points are given in Fig. 1 .

Fig. 2 illustrates how the proposed algorithm works in the case of three descriptions. The depicted index assignment is for three balanced descriptions computed for the sublattice of index $N=73$ that is generated by basis vectors: $8-\omega, \omega(8-\omega)$.

\subsection{Complexity}

The presented algorithm is fast with an $O(N)$ time complexity. The simplicity and low complexity of the presented index assignment are clear because we took a greedy optimization approach in designing it. The tantalizing question is, of course, can it be optimal? Let the distance between a nearest pair of central lattice points in $\Lambda$ be one. The result of [3] for the first example (best known so far) is $d_{s}=561 / 31=18.0968$, while the greedy algorithm does better, producing $d_{s}=528 / 31=17.0323$. Indeed, in both examples, one can verify that the expected distortion is minimized as the two terms of $d_{s}$ are minimized independently.

In the next section we will prove, under fairly relaxed conditions, that our greedy index assignment algorithm is optimal for two balanced descriptions despite its simplicity.

The only exact algorithm known so far for optimal MDLVQ index assignment is linear assignment. Although applying the linear assignment algorithm to optimize the index assignment is conceptually straightforward, a key issue that determines the complexity remains inadequately treated. This is how to reduce the labeling problem 
from an association between two infinite sets $\Lambda$ and $\Lambda_{s}^{K}$ to between two finite sets, and keep the latter two sets as small as possible without compromising optimality. A solution proposed by [4] seems to require $O\left(N^{6}\right)$ time because it used a candidate set of $O\left(N^{2}\right)$ central lattice points (the complexity of the linear assignment problem is cubic in the worst case). Even with such a large set of candidate central lattice points, still no bound was given on the size of the candidate $K$-tuples of sublattice points used for labeling, and no proof of optimality was offered.

\section{Proof of Optimality}

In the following development, we consider the natural construction: the sublattice Voronoi cell $V_{s}\left(\lambda_{s}\right)$ centered at $\lambda_{s} \in \Lambda_{s}$ contains the $N$ nearest central lattice points to $\lambda_{s}$. To prove the optimality of the greedy algorithm, we need some additional properties.

Lemma 1 If $\lambda \in V_{s / 2}(\tau)$ and $\tilde{\lambda} \notin V_{s / 2}(\tilde{\tau})$, where $\lambda, \tilde{\lambda} \in \Lambda$ and $\tau, \tilde{\tau} \in \Lambda_{s / 2}$, then $\|\lambda-\tau\| \leq\|\tilde{\lambda}-\tilde{\tau}\|$.

Proof: $\quad$ Scaling both $\lambda$ and $V_{s / 2}(\tau)$ by 2 places the lattice point $2 \lambda$ in $V_{s}(2 \tau)$; scaling both $\tilde{\lambda}$ and $V_{s / 2}(\tilde{\tau})$ by 2 places the lattice point $2 \tilde{\lambda} \notin V_{s}(2 \tilde{\tau})$. Since a sublattice Voronoi cell contains the nearest central lattice points, $\|2 \lambda-2 \tau\| \leq\|2 \tilde{\lambda}-2 \tilde{\tau}\|$, and hence $\|\lambda-\tau\| \leq\|\tilde{\lambda}-\tilde{\tau}\|$.

Definition $1 \mathrm{~A}$ lattice $\Lambda_{s}$ is said to be $S$-similar to $\Lambda$, if $\Lambda_{s}$ can be generated by scaling and rotating $\Lambda$ around any point $\tau \in \Lambda_{s / 2}$ and $\Lambda_{s}$ is a sublattice of $\Lambda$.

Note the S-similarity requires that the center of symmetry be a point in $\Lambda_{s / 2}$.

In what follows we assume that sublattice $\Lambda_{s}$ is $S$-similar to $\Lambda$. Also, we denote by $V_{\tau}$ the region created by scaling and rotating $V_{s / 2}(\tau)$ around $\tau$.

Lemma 2 If $\lambda_{s} \in V_{\tau}$ and $\tilde{\lambda}_{s} \notin V_{\tilde{\tau}}$, where $\lambda_{s}, \tilde{\lambda}_{s} \in \Lambda_{s}$ and $\tau, \tilde{\tau} \in \Lambda_{s / 2}$, then $\left\|\lambda_{s}-\tau\right\| \leq$ $\left\|\tilde{\lambda}_{s}-\tilde{\tau}\right\|$.

Proof: This lemma follows from Lemma 1 and the definition of $S$-Similar.

Lemma $3 \forall \tau \in \Lambda_{s / 2}$, the sublattice points in $V_{\tau}$ form $\left|\Lambda \cap V_{s / 2}(\tau)\right|$ nearest ordered 2-tuples with their midpoints being $\tau$.

Proof: Letting $\tilde{\tau}=\tau$ in Lemma 2, we see that $V_{\tau}$ contains the $\left|\Lambda_{s} \cap V_{\tau}\right|=$ $\left|\Lambda \cap V_{s / 2}(\tau)\right|$ nearest sublattice points to $\tau$. Thus this lemma follows from Property 3.

Theorem 1 The proposed greedy algorithm is optimal if the sublattice is S-Similar to its central lattice. 
Proof: By Property 1 , for any $\lambda_{1}, \lambda_{2} \in \Lambda_{s},\left(\lambda_{1}+\lambda_{2}\right) / 2 \in \Lambda_{s / 2}$. Now referring to (3), the proposed algorithm minimizes the second term $\sum_{\lambda \in \Lambda}\left\|\lambda-\left(\lambda_{1}+\lambda_{2}\right) / 2\right\|^{2} P(\lambda)$ of $d_{s}$, since it labels any central lattice point $\lambda \in V_{s / 2}(\tau)$ by $\left(\lambda_{1}, \lambda_{2}\right) \in \Lambda_{s}^{2}$, and $\left(\lambda_{1}+\lambda_{2}\right) / 2=\tau$. The first term $\sum_{\lambda \in \Lambda} \frac{1}{4}\left\|\lambda_{1}-\lambda_{2}\right\|^{2} P(\lambda)$ of $d_{s}$ is also independently minimized by the algorithm. Assume that $\sum_{\lambda \in \Lambda}\left\|\lambda_{1}-\lambda_{2}\right\|^{2} P(\lambda)$ was not minimized. Then there exists an ordered 2-tuple $\left(\tilde{\lambda}_{1}, \tilde{\lambda}_{2}\right) \in \Lambda_{s}^{2}$ which is not used in the index assignment, and $\left\|\tilde{\lambda}_{1}-\tilde{\lambda}_{2}\right\|<\left\|\lambda_{1}-\lambda_{2}\right\|$, where $\left(\lambda_{1}, \lambda_{2}\right) \in \Lambda_{s}^{2}$ is used in the index assignment. Let $\tau=\left(\lambda_{1}+\lambda_{2}\right) / 2, \tilde{\tau}=\left(\tilde{\lambda}_{1}+\tilde{\lambda}_{2}\right) / 2$. Since $\left(\lambda_{1}, \lambda_{2}\right)$ is used to label a central lattice point in $V_{s / 2}(\tau), \lambda_{1}, \lambda_{2} \in V_{\tau}$ by Lemma 3 . However, $\tilde{\lambda}_{1}, \tilde{\lambda}_{2} \notin V_{\tilde{\tau}}$, otherwise $\left(\tilde{\lambda}_{1}, \tilde{\lambda}_{2}\right)$ would be used in the index assignment by Lemma 3 . So we have $\left\|\lambda_{1}-\tau\right\| \leq\left\|\tilde{\lambda}_{1}-\tilde{\tau}\right\|$ by Lemma 2 , hence $\left\|\lambda_{1}-\lambda_{2}\right\| \leq\left\|\tilde{\lambda}_{1}-\tilde{\lambda}_{2}\right\|$, contradicting $\left\|\tilde{\lambda}_{1}-\tilde{\lambda}_{2}\right\|<\left\|\lambda_{1}-\lambda_{2}\right\|$.

The above proof of algorithm optimality requires the $S$-similarity of the sublattice. The following theorems show that $S$-similar sublattices can be easily found for many $N$ values, especially for many lattices commonly used in signal quantization, such as $A_{2}, Z^{2}, Z^{L}(L=4 k)$, and $Z^{L}$ ( $L$ odd). In the following statements, $m$ is a positive integer.

Theorem 2 An L-dimensional lattice $\Lambda$ has an $S$-similar sublattice with index $N$, if $N=m^{L}$ is odd.

Theorem 3 For the $A_{2}$ or $Z_{2}$ lattice $\Lambda$, a sublattice $\Lambda_{s}$ is $S$-similar to $\Lambda$, if it is geometrically similar to $\Lambda$ and clean.

Theorem 4 The $Z^{L}(L=4 l, l \geq 1)$ lattice $\Lambda$ has an $S$-similar, clean sublattice with index $N$, if $N=m^{L / 2}$ with $m=4 n+1$.

Theorem 5 The $Z^{L}$ (L is odd) lattice $\Lambda$ has an $S$-similar, clean sublattice with index $N$, if and only if $N=m^{L}$ is odd.

\section{Analytical Results for Two Descriptions and Op- timal $\mathbf{N}$}

Up to now no closed form expression is known for the expected distortion of optimal MDLVQ, except for an asymptotic result as $N \rightarrow \infty$ [3]. However, practical scenarios for MDLVQ usually involve modest values of $N$ [5]. As a byproduct of our algorithm development and the proof of its optimality, we can now derive the closed form analytical solution of the side distortion achieved by optimal MDLVQ for two balanced descriptions. Under high resolution assumption,

$$
d_{c}=G_{\Lambda} \nu^{2 / L}
$$

where $\nu$ is the $L$-dimensional volume of a Voronoi region of $\Lambda$, and $G_{\Lambda}$ is the dimensionless normalized second moment of lattice $\Lambda$. 
Let $a_{i}$ be the squared distance of the $i^{\text {th }}$ nearest sublattice point to the origin, and $b_{i}$ be the squared distance of the $i^{t h}$ nearest central lattice point in $V_{s}(0)$ to the origin. We can show that the two terms in $d_{s}$ are

$$
\sum_{\lambda \in \Lambda} \frac{1}{4}\left\|\lambda_{1}-\lambda_{2}\right\|^{2} P(\lambda)=\frac{1}{4 L} \frac{\sum_{i=1}^{N} a_{i}}{N}, \quad \sum_{\lambda \in \Lambda}\left\|\lambda-m_{1,2}\right\|^{2} P(\lambda)=\frac{1}{4 L} \frac{\sum_{i=1}^{N} b_{i}}{N} .
$$

The above equations lead to some interesting observations. The term $N^{-1} \sum_{i=1}^{N} a_{i}$ is the average squared distance of the $N$ nearest sublattice points to the origin, which was also realized by previous authors [3]. The other term $N^{-1} \sum_{i=1}^{N} b_{i}$ is the average squared distance of central lattice points in $V_{s}(0)$ to the origin.

Substituting (4) and (5) into (1), we get the closed form expression for the expected distortion,

$$
D=\left(1-p_{l}^{2}\right) G_{\Lambda} \nu^{2 / L}+\frac{1}{2} p_{l}\left(1-p_{l}\right) L^{-1} N^{-1}\left(\sum_{i=1}^{N} a_{i}+\sum_{i=1}^{N} b_{i}\right)+p_{l}^{2} E\left[\|X\|^{2}\right],
$$

Let $h(p)$ be the source entropy rate per dimension, then the entropy rate of a side description is given by [3],

$$
R=h(p)-(1 / L) \log _{2}(N \nu) .
$$

Thus the optimal $N$ can be solved by combing (6) and (7), rather than solving many instances of index assignment problem for varying $N$.

For $Z$ lattice, (6) can be simplified to

$$
D=\left(1-p_{l}^{2}\right) G_{\Lambda} \nu^{2 / L}+\frac{1}{24} p_{l}\left(1-p_{l}\right)\left(N^{4}-1\right) \nu^{2 / L}+p_{l}^{2} E\left[\|X\|^{2}\right], \quad i=1,2 .
$$

\section{Analysis for $K \geq 2$ Descriptions and Optimal $N$, $K$}

Now we derive a closed form expression of the expected distortion of optimal MDLVQ for general $K \geq 2$ asymptotically. Our result improves a similar formula presented in [4] that includes an empirically determined constant. It also allows us to determine the optimal sublattice index $N$, as well as the optimal number of descriptions $K$.

Let $N_{\tau}$ denote the number of central lattice points in $V_{s / K}(\tau)$. As $N \rightarrow \infty$, each Voronoi cell of $\Lambda_{s / K}$ contains approximately $N_{\tau} \approx N / K^{L}$ central lattice points, which are uniformly distributed $V_{s / K}(\tau)$. Hence the second term of $d_{s}$ is

$$
\frac{\zeta_{1}}{\zeta_{2}} \sum_{\lambda \in \Lambda}\left\|\lambda-m_{K}\right\|^{2} P(\lambda)=\frac{\zeta_{1}}{\zeta_{2}} G_{\Lambda}\left(N_{\tau} \nu\right)^{2 / L}=\frac{\zeta_{1}}{\zeta_{2}} G_{\Lambda} K^{-2}(N \nu)^{2 / L}
$$

To analyze the first term of $d_{s}$, we evaluate $\sum_{k=1}^{K}\left\|\lambda_{k}-m_{K}\right\|^{2}$ for the $N_{\tau}$ nearest sublattice $K$-tuples $\left(\lambda_{1}, \lambda_{2}, \cdots, \lambda_{K}\right)$ with centroids $m_{K}=\tau \in \Lambda_{s / K}$. Let

$$
f \equiv \sum_{k=1}^{K}\left\|\lambda_{k}-m_{K}\right\|^{2}, \quad \varsigma_{k} \equiv \sum_{i=1}^{k} \lambda_{i}, k=1,2, \cdots, K
$$


After some tedious algebra

$$
f=\sum_{k=1}^{K-1} \frac{k+1}{k}\left\|\varsigma_{k}-\frac{k}{k+1} \varsigma_{k+1}\right\|^{2}
$$

There is a one-to-one correspondence between $\left(\lambda_{1}, \lambda_{2}, \cdots, \lambda_{K}\right)$ and $\left(\varsigma_{1}, \varsigma_{2}, \cdots, \varsigma_{K}\right)$. So the problem of finding $N_{\tau}$ nearest sublattice $K$-tuples $\left(\lambda_{1}, \lambda_{2}, \cdots, \lambda_{K}\right)$ with given centroid $m_{K}=\tau \in \Lambda_{s / K}$, is equivalent to finding the $N_{\tau}$ optimal sublattice $(K-1)$ tuples $\left(\varsigma_{1}, \varsigma_{2}, \cdots, \varsigma_{K-1}\right)$ given $\varsigma_{K}=K m_{K}$ in (9).

The $i^{\text {th }}$ nearest sublattice points to $\frac{k}{k+1} \varsigma_{k+1}$ is approximately on the boundary of an $L$ dimensional sphere with volume $i N \nu$. So given $\varsigma_{k+1}$, the $i^{\text {th }}$ smallest value of $\left\|\varsigma_{k}-\frac{k}{k+1} \varsigma_{k+1}\right\|^{2}$ is approximately $\left(i N \nu / V_{L}\right)^{2 / L} / L=G_{L}(L+2)(i N \nu)^{2 / L} / L$, where $V_{L}$ is the volume of an $L$-dimensional sphere of unit radius, and $G_{L}$ is the dimensionless normalized second moment of an $L$-dimensional sphere.

Let $f$ take on its $n^{\text {th }}$ smallest value $f^{(n)}$ at $\left(\varsigma_{1}^{(n)}, \varsigma_{2}^{(n)}, \cdots, \varsigma_{K-1}^{(n)}\right)$, and let the sum $\sum_{k=1}^{K-1} \frac{k+1}{k}\left(i_{k}\right)^{2 / L}$ take on its $n^{t h}$ smallest value at $\left(i_{1}^{(n)}, i_{2}^{(n)}, \cdots, i_{K-1}^{(n)}\right) \in \mathbb{Z}^{K-1}$. Then

$$
f^{(n)}=\sum_{k=1}^{K-1} \frac{k+1}{k}\left\|\varsigma_{k}^{(n)}-\frac{k}{k+1} \varsigma_{k+1}^{(n)}\right\|^{2} \approx\left(1+\frac{2}{L}\right) G_{L}(N \nu)^{2 / L} \sum_{k=1}^{K-1} \frac{k+1}{k}\left(i_{k}^{(n)}\right)^{2 / L} .
$$

Hence,

$$
\sum_{\lambda \in \Lambda} \sum_{k=1}^{K}\left\|\lambda_{k}-m_{K}\right\|^{2} P(\lambda) \approx\left(1+\frac{2}{L}\right) G_{L}(N \nu)^{2 / L} \frac{1}{N_{\tau}} \sum_{n=1}^{N_{\tau}} \sum_{k=1}^{K-1} \frac{k+1}{k}\left(i_{k}^{(n)}\right)^{2 / L}
$$

After lengthy derivation (omitted due to space limit), we arrive at

$$
\frac{1}{K} \sum_{\lambda \in \Lambda} \sum_{k=1}^{K}\left\|\lambda_{k}-m_{K}\right\|^{2} P(\lambda) \approx G_{L} \Phi_{K-1, L}(K-1) K^{\frac{-K}{K-1}} N^{\frac{K}{K-1}} \frac{2}{L} \nu^{\frac{2}{L}}
$$

where

$$
\Phi_{n, L}=\frac{1+\frac{2}{L}}{n+\frac{2}{L}} \frac{\Gamma\left(\frac{n L}{2}+1\right)^{\frac{2}{n L}}}{\Gamma\left(\frac{L}{2}+1\right)^{\frac{2}{L}}} .
$$

Note $\Phi_{K-1, L}=12 G_{K-1}$ for $L=1$, and $\Phi_{K-1, L}=1$ for $K=2$.

Comparing (8) with (10), the first term of $d_{s}$ dominates the second term when $N \rightarrow \infty$, thus

$$
d_{s} \approx G_{L} \Phi_{K-1, L}(K-1) K^{\frac{-K}{K-1}} N^{\frac{K}{K-1}} \frac{2}{L} \nu^{\frac{2}{L}} .
$$

Substituting (11) and (4) into (1), we finally get a closed form solution of the expected distortion for optimal MDLVQ

$$
D=\left(1-p_{l}^{K}\right) G_{\Lambda} \nu^{2 / L}+\zeta_{2} G_{L} \Phi_{K-1, L}(K-1) K^{\frac{-K}{K-1}} N^{\frac{K}{K-1} \frac{2}{L}} \nu^{\frac{2}{L}}+p_{l}^{K} E\left[\|X\|^{2}\right] .
$$

The total target entropy is $R_{t}=K R$, so we rewrite (7) to get

$$
N \nu=2^{L\left(h(X)-R_{t} / K\right)}=\eta,
$$


where $\eta$ is constant. Now we have

$$
D=\left(1-p_{l}^{K}\right) G_{\Lambda} \nu^{2 / L}+\zeta_{2} G_{L} \Phi_{K-1, L}(K-1) K^{\frac{-K}{K-1}} \eta^{\frac{K}{K-1} \frac{2}{L}} \nu^{\frac{-1}{K-1} \frac{2}{L}}+p_{l}^{K} E\left[\|X\|^{2}\right] .
$$

By differentiating $D$ to $\nu$, we get the optimal $\nu$. Substituting it to (13), we get optimal $N$ :

$\nu_{o p t}=\eta\left(\frac{\zeta_{2}}{1-p_{l}^{K}} \frac{G_{L}}{G_{\Lambda}} K^{-\frac{K}{K-1}} \Phi_{K-1, L}\right)^{\frac{L(K-1)}{2 K}}, \quad N_{o p t}=\left(\frac{1-p_{l}^{K}}{\zeta_{2}} \frac{G_{\Lambda}}{G_{L}} K^{\frac{K}{K-1}} \frac{1}{\Phi_{K-1, L}}\right)^{\frac{L(K-1)}{2 K}}$.

Note $N_{\text {opt }}$ is independent of the total target entropy rate $R_{t}$ and source entropy rate $h(p)$. It only depends on the loss probability $p_{l}$ and on the number of descriptions $K$. Substituting $\nu_{\text {opt }}$ into (14), the average distortion can be expressed as a function of $K$. Then optimal $K$ can be solved numerically.

\section{Conclusions}

Although optimal MDLVQ index assignment is conceptually a problem of linear assignment, the problem is originally about finding a bijective mapping between two infinite sets $\Lambda$ and $\Lambda_{s}^{K}$. No good solutions are known to reduce the underlying bipartite graph to a modest size without missing optimality. Therefore, the problem remains largely open. We presented a linear-time algorithm for solving the problem of optimal MDLVQ index assignment. The algorithm is proven to be optimal for two balanced descriptions in any dimensions. We conjecture that the algorithm is also optimal for any number of balanced descriptions.

We also made progress in the analysis of MDLVQ performance. Exact closed form expression of the expected distortion was derived for $K=2$ and for any $N$. For cases $K>2$, we improved the current asymptotic expression of the expected distortion.

\section{References}

[1] J. H. Conway and N. J. A. Sloane, "Sphere Packings, Lattices, and Groups," 3rd edition, Springer, 1998.

[2] S. D. Servetto, V. A. Vaishampayan, and N. J. .A. Sloane, "Multiple description lattice vector quantization," in Proc. Data Compression Conf., pp. 13-22, Mar. 1999.

[3] V. A. Vaishampayan, N. J. A. Sloane, and S. D. Servetto, "Multipledescription vector quantization with lattice codebooks: Design and analysis," IEEE Trans. Inform. Theory, vol. 47, no. 5, pp. 1718-1734, July 2001.

[4] J. Østergaard, J. Jensen and R. Heusdens, " $n$-channel Symmetric Multiple-Description Lattice Vector Quantization," in Proc. Data Compression Conf., pp. 378-387, Mar. 2005.

[5] V. K. Goyal, J. A. Kelner and J. Kovačević, "Multiple description vector quantization with a coarse lattice," IEEE Trans. Inform. Theory, vol. 48, pp. 781-788, Mar. 2002. 